\title{
Calf Circumference as an Optimal Choice of Four Screening Tools for Sarcopenia Among Ethnic Chinese Older Adults in Assisted Living
}

This article was published in the following Dove Press journal: Clinical Interventions in Aging

\author{
Chung-Yao Chen $\mathbb{D}^{1,2}$ \\ Wen-Chun Tseng' \\ Yao-Hung Yang ${ }^{\prime}$ \\ Chia-Ling Chen ${ }^{3,4}$ \\ Lain-Li Lin $^{5}$ \\ Fang-Ping Chen ${ }^{2,6,7}$ \\ Alice MK Wong ${ }^{2,3,8}$
}

'Department of Physical Medicine and Rehabilitation, Chang Gung Memorial Hospital, Keelung 204, Taiwan;

${ }^{2}$ Department of Medicine, College of Medicine, Chang Gung University, Taoyuan 333, Taiwan; ${ }^{3}$ Department of Physical Medicine and Rehabilitation, Chang Gung Memorial Hospital, Linkou 333, Taiwan; ${ }^{4}$ Graduate Institute of Early Intervention, College of Medicine, Chang Gung University, Taoyuan 333, Taiwan; ${ }^{5}$ Department of Tourism Leisure and Health, Ching Kuo Institute of Management and Health, Keelung 204, Taiwan; ${ }^{6}$ Keelung Osteoporosis

Prevention and Treatment Center, Chang Gung Memorial Hospital, Keelung 204,

Taiwan; ${ }^{7}$ Department of Obstetrics and Gynecology, Chang Gung Memorial Hospital, Keelung 204, Taiwan; ${ }^{8}$ Healthy Aging Research Center, Chang Gung University, Taoyuan 333, Taiwan
Introduction: Sarcopenia is highly prevalent among residents of assisted-living facilities. However, the optimal screening tools are not clear. Therefore, we compared the performance of four recommended screening tools for predicting sarcopenia.

Methods: The study recruited 177 people over 65 years of age in assisted-living facilities. Appendicular muscle mass index was measured using bioelectrical impedance analysis. Calf circumference (CC), handgrip, six-meters walking speed, and screening questionnaires including SARC-CalF, SARC-F and 5-item Mini Sarcopenia Risk Assessment (MSRA-5) were evaluated. The diagnosis criteria for sarcopenia were based on the Asian Working Group for Sarcopenia 2019 consensus. The area under the receiver operating characteristic curves (AUC) was used to contrast the diagnostic accuracy of screening tools.

Results: The prevalence of sarcopenia was $52.7 \%$ among men and $51.2 \%$ among women. After adjusting for age, sex, body mass index and SARC-CalF score, CC remained significantly associated with sarcopenia in logistic regression analysis. The prediction model for sarcopenia based on $\mathrm{CC}$ alone had the highest accuracy compared to SARC-CalF, MSRA-5 and SARC-F (AUC, 0.819 vs 0.734 vs 0.600 vs 0.576 ; sensitivity/specificity, $80.4 \% / 71.8 \%$ vs $38.0 \% / 80.0 \%$ vs $60.7 \% / 54.2 \%$ vs $10.9 \% / 91.8 \%$ ). Differences in AUCs between the prediction models were statistically significant (CC vs. SARC-CalF, P $=0.0181$; SARCCalF vs. MSRA-5, $\mathrm{P}=0.0042)$. Optimal cutoff values for predicting sarcopenia were CC $<34 \mathrm{~cm}$ in men and $<33 \mathrm{~cm}$ in women.

Conclusion: To predict sarcopenia based on low CC alone is accurate, easy and inexpensive for use in assisted-living facility settings. Further validation studies in different populations are suggested.

Keywords: sarcopenia, calf circumference, SARC-F, SARC-CalF, MSRA, assisted-living

\section{Introduction}

Taiwan officially became an aging society in 2018 and within years will face becoming a super-aged society. Sarcopenia, a prominent issue in the field of geriatrics, refers to low muscle mass, low muscle strength, and low physical performance associated with aging. Several sarcopenia study groups have established different diagnostic criteria for sarcopenia. $^{1,2}$ The prevalence of sarcopenia ranges from $5 \%$ to $13 \%$ in adults ages $60-70$ years, rising to $50 \%$ in people over 80 years old. ${ }^{3}$ Several studies have revealed that sarcopenia leads to deterioration of activities of daily living, ${ }^{4}$ physical function, ${ }^{5}$ and increased mortality ${ }^{6}$ in geriatric populations. Because diagnosing sarcopenia requires special instruments and is time- and resource-consuming,
Correspondence: Chung-Yao Chen Department of Physical Medicine and Rehabilitation, Chang Gung Memorial Hospital, No. 200, Ln. 208, Jijin Ist Road, Anle District, Keelung City 204, Taiwan Tel +886-2-2432-9292 \#2725

Email jongyau2002@gmail.com 
simple and effective screening tools for sarcopenia are needed for geriatric populations.

Several tools are available to screen for sarcopenia. The updated consensus of the EWGSOP (European Working Group on Sarcopenia in Older People) and the AWGS (Asian Working Group for Sarcopenia) recommended SARC-F (strength, assistance with walking, rise from a chair, climb stairs and falls) as a screening tool in primary health care or community preventive care settings. ${ }^{1,2}$ However, several studies have reported high specificity but poor sensitivity for $\mathrm{SARC}-\mathrm{F}^{7,8}$; these remain pitfalls for early disease detection. A systematic review and meta-analysis study had found that the average prevalence of sarcopenia in nursing homes was $41 \%{ }^{9}$ As exercise and diet control programs are often available and mandatory in long-term care facilities, it is important to have effective methods for selecting those at risk of sarcopenia to participate in exercise and diet programs. Therefore, sensitive and accurate screening tools for sarcopenia are important in long-term care settings. Other questionnaires, such as the 5-item Mini Sarcopenia Risk Assessment (MSRA-5), have been developed to screen for sarcopenia, increasing the sensitivity up to $80.4 \%$, but the new instrument had decreased specificity $(60.4 \%){ }^{10}$

Anthropometric measurement is a cheap and easily available method to estimate sarcopenia. The EWGSOP consensus in 2010 and 2018 did not recommend calf circumference (CC) as a screening tool because of the wide variation in $\mathrm{CC}$ due to age-related changes in fat deposits and decreased skin elasticity. ${ }^{1,11}$ Given that adipose tissue accumulates more easily over the abdomen than the extremities in Chinese and South Asian people as compared to Europeans, ${ }^{12}$ it is plausible that $\mathrm{CC}$ is less likely to be influenced by adipose tissue among Asians. Growing evidence has suggested that $\mathrm{CC}$ is associated with sarcopenia or low muscle mass in Asians ${ }^{13-16}$ and the AWGS 2019 consensus recommended use of $\mathrm{CC}$ for case-finding. ${ }^{2}$ Moreover, a research group from Brazil developed a combined scoring method by adding $\mathrm{CC}$ to the SARC-F; the resulting SARC-CalF model of screening for sarcopenia significantly improved the sensitivity of SARC-F from $33 \%$ to $67 \%$ without weakening its specificity. ${ }^{17}$ The performance of SARC-CalF has been evaluated in community-dwelling ${ }^{17,18}$ and nursing home older adults. ${ }^{19,20}$ In particular, a study in Slovenia showed this method had sensitivity of up to $77.4 \%$ and specificity of up to $89.8 \%$ for predicting sarcopenia using the EWGSOP criteria. ${ }^{19}$
Although the AWGS 2019 consensus recommended CC to screen for sarcopenia, the performance of CC alone for predicting sarcopenia has not been directly compared to other popular screening tools. This cross-sectional study was consequently designed to evaluate the performance of CC, MSRA5, SARC-F and SARC-Calf models for predicting sarcopenia among older ethnic Chinese adults in assisted-living facilities.

\section{Materials and Methods Study Design and Population}

The study recruited residents over 65 years old residing in two assisted living facilities in northern Taiwan. Exclusion criteria included the presence of bilateral pitting edema of the legs greater than grade 2, dementia or cognitive impairment by the Mini-Cog test, ${ }^{21}$ loss of ability to walk independently, and contraindications for bioelectrical impedance analysis (BIA) such as having an implantable pacemaker.

Sarcopenia was diagnosed based on the AWGS 2019 criteria, ${ }^{2}$ which combines low muscle mass (using the appendicular skeletal muscle index, ASMI: men $<7.0 \mathrm{~kg} /$ $\mathrm{m}^{2}$ and women $<5.7 \mathrm{~kg} / \mathrm{m}^{2}$ ) with low grip strength (men $<28 \mathrm{~kg}$, women $<18 \mathrm{~kg})$ or low walking speed $(<1.0 \mathrm{~m} / \mathrm{s})$. A qualified physiotherapist completed measurement of skeletal muscle mass, grip strength, calf circumference, and questionnaire. The study was conducted in accordance with the Declaration of Helsinki and the Institutional Review Board of Chang Gung Medical Foundation approved the study design protocol (IRB number: 201800398A3). Written informed consent was obtained from all participants and/or their legal guardians.

\section{Measurement of Appendicular Skeletal Muscle Mass}

Muscle mass and body fat mass was measured with a multi-frequency segmental BIA device (Inbody 270, InBody Co, South Korea). All participants were asked to fast for 8 hours and were measured by BIA in the morning after urination. Appendicular skeletal muscle mass (ASM) was computed as the sum of the muscle mass of the four limbs, then normalized by height squared to yield appendicular skeletal mass index (ASMI, $\mathrm{kg} / \mathrm{m}^{2}$ ).

\section{Measurement of Grip Strength, Usual Walking Speed, and Other Anthropometric Measures}

Grip strength was measured with the Handgrip Dynamometer (GT-315, OG Wellness Technologies Co, 
Japan) in a sitting position with elbow flexion at $90^{\circ}$. Three trials were done for each hand and the mean value of six trials was taken to represent handgrip strength. ${ }^{22}$ Measurement of the sound hand instead of the paretic hand was performed in participants with stroke. Walking speed was measured by asking participants to walk a six-meter distance at their usual speed without assistance, starting from a static standing position. ${ }^{22}$ After sitting for 15 minutes, the mean $\mathrm{CC}$ was measured at the widest level of both calves in a seated and relaxed position with knee flexion at $90^{\circ}$ using inextensible tape. ${ }^{23}$ If prominent leg edema or hemiplegia was noted, the contralateral calf was measured instead. Body height and weight were measured and body mass index (BMI) was calculated.

\section{Screening Tools: SARC-F, SARC-CalF and the MSRA-5 Questionnaire}

The standard SARC-F tool consists of a five-item questionnaire, which includes strength, assistance in walking, rising from chair, climbing stairs and falls. ${ }^{8}$ Each item received a score from 0 to 2 points. A total score of $\geq 4$ indicates sarcopenia. ${ }^{8}$

The SARC-CalF ${ }^{17}$ was comprised of the standard fiveitem SARC-F and CC. The CC score was 0 if $\mathrm{CC}$ was above the cutoff value $(34 \mathrm{~cm}$ for men and $33 \mathrm{~cm}$ for women) and 10 points if $\mathrm{CC}$ was below the cutoff value, based on the recommendations of the AWGS 2019 consensus. ${ }^{2}$ A total score of $\geq 11$ indicated sarcopenia. ${ }^{2}$

The MSRA-5 questionnaire also consists of five yes or no items including age, hospitalization, activity, regular meals, and weight loss. ${ }^{10}$ Scores for each question range from 0 to 15 points, and a total score of $\leq 45$ indicates sarcopenia. ${ }^{10}$ The Chinese version of the SARC-F and MSRA-5 have been previously validated. ${ }^{24,25}$

\section{Statistical Analysis}

Normality of data distribution was assessed using the Shapiro-Wilk test. Categorical data were reported as numbers (\%) and continuous variables were reported as means \pm standard deviation or medians (interquartile range, IQR). In order to distinguish those with and without sarcopenia, categorical variables were compared using a Chi-square test or Yate's continuity correlation, and continuous variables were compared using the unpaired $t$-test or the Mann-Whitney $U$ test. Except grip strength, walking speed and ASMI, variables were selected for inclusion in the multivariate logistic regression model if they were significantly related to sarcopenia $(p<0.05)$ in bivariate analyses to determine which were independent predictors for sarcopenia. We also calculated the sensitivity, specificity, positive likelihood ratio, and negative likelihood ratio for the SARC-CalF, SARC-F, MSRA-5 and CC models. Receiver operating characteristics (ROC) analysis was performed for the CC, SARC-F, MSRA-5, and SARCCalF models, calculating the area under the ROC curve (AUC) and 95\% confidence intervals. We compared the AUC of the different ROC curves for predicting sarcopenia, using the DeLong method. ${ }^{26}$ Based on type I error of 0.05 and type II error of 0.20 , the reported AUC of $\mathrm{CC}^{15}$ and SARC-CalF, ${ }^{17}$ the correlation in positive and negative group of 0.6 , and the ratio of sample sizes in positive/ negative groups of 1 according to our preliminary data, the required total sample size of 164 was calculated. In order to determine the optimal $\mathrm{CC}$ cutoff value for predicting sarcopenia, subgroup analysis stratified by sex was performed using ROC analysis and the Youden index. ${ }^{27}$ Statistical analysis was performed using MedCalc (version 19.5.3; MedCalc Software, Ostend, Belgium) for ROC analysis and SPSS (version 22.0; SPSS Statistics; IBM, Armonk, NY).

\section{Results}

The study screened 236 older adults in two assisted living facilities and recruited 93 men and 84 women, with a mean age of $78.7 \pm 8.6$ years for men and $81.1 \pm 6.8$ years for women. Thirty adults refused to join the study and 29 adults were excluded due to cognitive impairment $(\mathrm{n}=13)$, impaired walking ability $(\mathrm{n}=7)$ and contraindications for BIA including cardiac devices $(\mathrm{n}=4)$ and metal implants $(n=5)$, respectively. Demographic characteristics of the recruited participants are listed in Table 1 . The prevalence of sarcopenia was $52.7 \%$ among the men and $51.2 \%$ among the women. There was no significant difference in underlying disease between participants with and without sarcopenia. Participants with sarcopenia had significantly higher SARCCalF scores and lower BMI, grip strength, and ASMI scores, as well as lower CC measurements compared to participants without sarcopenia (Table 1). Among the women, patients with sarcopenia were significantly older than those without. Therefore, an age-by-sex interaction term was entered into the logistic regression model. After entering the variables of age, sex, BMI, CC and SARC-CalF scores along with the two-way age-by-sex interaction term into the equation, binary logistic regression analysis revealed that BMI, SARC$\mathrm{CalF}$ scores and $\mathrm{CC}$ were significantly associated with 
Table I Basic Characteristics, Comorbidity, Sarcopenia Markers, and Scores from Screening Tools

\begin{tabular}{|c|c|c|c|c|c|c|}
\hline & \multicolumn{3}{|l|}{ Men $(\mathrm{N}=93)$} & \multicolumn{3}{|l|}{ Women $(\mathrm{N}=84)$} \\
\hline & $\begin{array}{l}\text { Sarcopenia } \\
(N=49)\end{array}$ & $\begin{array}{l}\text { Non-Sarcopenia } \\
(\mathrm{N}=44)\end{array}$ & P-value & $\begin{array}{l}\text { Sarcopenia } \\
(\mathrm{N}=43)\end{array}$ & $\begin{array}{l}\text { Non-Sarcopenia } \\
(\mathrm{N}=4 \mathrm{I})\end{array}$ & P-value \\
\hline Age (year) & $79.6 \pm 8.5$ & $77.7 \pm 8.7$ & 0.278 & $82.9 \pm 5.4$ & $79.3 \pm 7.7$ & 0.015 \\
\hline BMI $\left(\mathrm{kg} / \mathrm{m}^{2}\right)$ & $22.4(20.4-23.8)$ & $25.6(23.6-28.7)$ & $<0.001$ & $21.2 \pm 3.3$ & $26.2 \pm 4.0$ & $<0.001$ \\
\hline Grip strength (kg) & $19.0(13.4-22.6)$ & $21.3(14.7-25.4)$ & 0.117 & $11.0(9.0-13.7)$ & $14.6(11.1-19.4)$ & 0.004 \\
\hline Gait speed $(\mathrm{m} / \mathrm{s})$ & $0.70 \pm 0.30$ & $0.80 \pm 0.30$ & 0.526 & $0.70 \pm 0.30$ & $0.70 \pm 0.30$ & 0.930 \\
\hline ASMI $\left(\mathrm{kg} / \mathrm{m}^{2}\right)$ & $6.20(5.85-6.70)$ & $7.40(7.13-7.78)$ & $<0.001$ & $4.90(4.60-5.40)$ & $6.4(5.95-6.70)$ & $<0.001$ \\
\hline HTN & $32(65.3 \%)$ & $33(75.0 \%)$ & 0.309 & $29(67.4 \%)$ & 31 (75.6\%) & 0.407 \\
\hline DM & $8(16.3 \%)$ & $10(22.7 \%)$ & 0.435 & $10(23.3 \%)$ & 14 (34.1\%) & 0.194 \\
\hline CAD & $9(18.4 \%)$ & $8(18.2 \%)$ & 0.982 & $8(18.6 \%)$ & $6(14.6 \%)$ & 0.625 \\
\hline Cancer & I (2.0\%) & I (2.3\%) & 1.000 & $3(7.0 \%)$ & $3(7.3 \%)$ & 1.000 \\
\hline Stroke & 7 (14.3\%) & $6(13.6 \%)$ & 0.928 & $3(7.0 \%)$ & $6(14.6 \%)$ & 0.435 \\
\hline$C C(\mathrm{~cm})$ & $31.5(30.0-34.0)$ & $34.5(33.5-37.9)$ & $<0.001$ & $29.5(26.6-31.2)$ & $34.0(31.5-35.4)$ & $<0.001$ \\
\hline CC below cutoff value* & $36(73.5 \%)$ & $12(27.3 \%)$ & $<0.001$ & $38(88.4 \%)$ & $14(34.1 \%)$ & $<0.001$ \\
\hline SARC-F score & I (0-3) & I $(0-2)$ & 0.263 & I (I-3) & I $(0-2)$ & 0.142 \\
\hline SARC-F (score $\geqq 4)$ & $9(18.4 \%)$ & $6(13.6 \%)$ & 0.536 & $6(14.0 \%)$ & $5(12.2 \%)$ & 0.811 \\
\hline MSRA-5 score & $40(40-55)$ & $50(40-55)$ & 0.078 & $40(32.5-55)$ & $50(35-55)$ & 0.116 \\
\hline MSRA-5 (score $\leqq 45)$ & $28(57.1 \%)$ & $19(43.2 \%)$ & 0.178 & $26(60.5 \%)$ & $19(46.3 \%)$ & 0.150 \\
\hline SARC-CalF score & $10(10-12)$ & $4.5(0-11)$ & 0.003 & $11(10-13)$ & $2(0-10.5)$ & $<0.001$ \\
\hline SARC-CalF (score $\geqq I I)$ & $23(46.9 \%)$ & $13(29.5 \%)$ & 0.086 & $31(72.1 \%)$ & $10(24.4 \%)$ & $<0.001$ \\
\hline
\end{tabular}

Notes: *Cutoff value: men $<34 \mathrm{~cm}$; women $<33 \mathrm{~cm}$. Data are mean \pm SD for normal distribution data and Median (Interquartile range) for non-normal distribution data, unless other specified.

Abbreviations: BMI, body mass index; ASMI, appendicular skeletal muscle index; DM, diabetic mellitus; HTN, hypertension; CAD, coronary artery disease; CC, calf circumference; MSRA-5, 5-item Mini Sarcopenia Risk Assessment.

sarcopenia (Table 2). The ROC curves and performance of the SARC-F questionnaire, the MSRA-5 questionnaire, the SARC-CalF and CC models in predicting sarcopenia are presented in Figure 1 and Table 3. Larger AUC values indicate better diagnostic accuracy of the screening tools, and the CC model had the largest AUC $(0.819,95 \% \mathrm{CI}$ 0.754-0.872) of the four screening tools. The $\mathrm{CC}$ model presented a significantly larger AUC than the SARC-CalF (AUC 0.819 vs 0.734, difference 0.085, 95\% CI $0.014-0.154, Z=2.364, \mathrm{P}=0.0181$ ), and the AUC of the SARC-CalF was significantly larger than that of the MSRA5 (AUC 0.734 vs 0.600, difference 0.139 , 95\% CI
0.044-0.234, $Z=2.863, \mathrm{P}=0.0042$ ) and SARC-F (AUC 0.734 vs 0.576 , difference 0.158 , 95\% CI $0.085-0.231$, $Z=4.239, \mathrm{P}<0.0001)$. The optimal cutoff values for predicting sarcopenia using $\mathrm{CC}$ were $<34 \mathrm{~cm}$ in men (sensitivity $73.5 \%$, specificity $72.7 \%$ ) and $<33 \mathrm{~cm}$ in women (sensitivity $88.4 \%$, specificity $65.9 \%$ ).

\section{Discussion}

Calf circumstance had best accuracy among four screening tools for predicting sarcopenia and was independently associated with sarcopenia after adjusting for SARCCalF score, sex, age, and BMI. The CC measurement

Table 2 Logistic Regression Analysis of Factors Associated with Sarcopenia

\begin{tabular}{|l|l|l|l|l|l|}
\hline Variable & $\boldsymbol{\beta}$ & SE & Exp(B) & 95\% CI & P-value \\
\hline Age & 0.041 & 0.032 & 1.042 & $0.978-1.11$ & 0.200 \\
Sex (women = 0, men = I) & -2.64 & 4.622 & 0.071 & $0-613.778$ & 0.568 \\
BMI & -0.34 & 0.078 & 0.712 & $0.611-0.83$ & $<0.001$ \\
SARC-CalF score & 0.091 & 0.044 & 1.096 & $1.004-1.195$ & 0.040 \\
CC & -0.17 & 0.080 & 0.884 & $0.772-0.987$ & 0.033 \\
Age by Sex interaction & 0.025 & 0.058 & 1.025 & $0.916-1.148$ & 0.668 \\
\hline
\end{tabular}

Notes: Variables were selected for inclusion in the multivariate logistic regression model if they were significantly related to sarcopenia $(p<0.05)$ in bivariate analyses. Cox \& Snell $R^{2}=0.403$, Nagelkerke $R^{2}=0.537$.

Abbreviations: BMI, body mass index; CC, calf circumference; SE, standard error; Cl, confidence interval. 

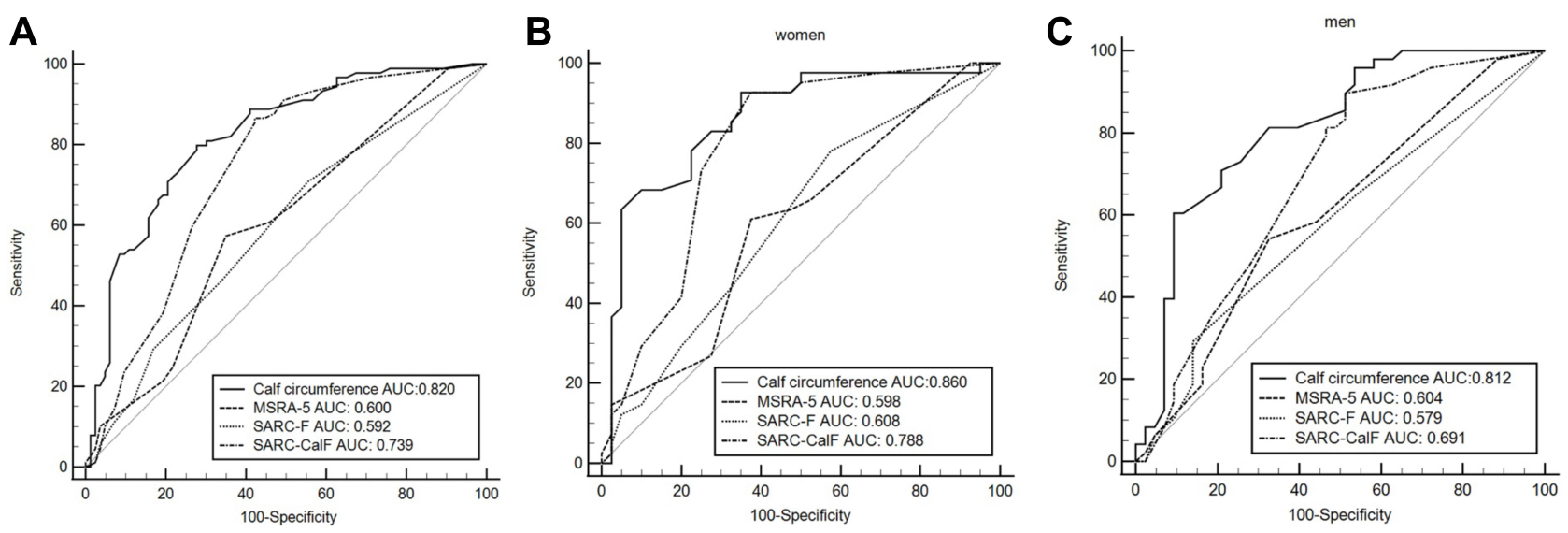

Figure I Receiver operating characteristic (ROC) curve of SARC-F, MSRA-5, SARC-CalF and Calf circumstance against the Criteria of Asian Working Group for Sarcopenia 2019 in (A) the total participants; (B) women and (C) men.

offers an accurate and easy-to-use screening tool for sarcopenia in assisted-living facilities.

Over half of the participants residing in assisted-living facilities were found to have sarcopenia according to the AWGS 2019 criteria. A meta-analysis study reported a higher prevalence of sarcopenia among nursing home residents, with a pooled prevalence of $41 \%(95 \% \mathrm{CI}$ $32-51 \%) .{ }^{9}$ The prevalence of sarcopenia observed in this study (52\%) is slightly above the range previously reported among nursing home residents and may be related to the new AWGS 2019 diagnostic criteria used in this study. The prevalence of a disease can affect the sensitivity and specificity, ${ }^{28}$ but not the $\mathrm{AUC}^{29}$ of a screening tool. The finding that specificity tends to decrease with higher disease prevalence $\mathrm{e}^{30}$ might explain why the specificity of the tests in this study was lower than that found in Chinese nursing homes ${ }^{20}$ (SARC-CalF, $80.0 \%$ vs $85.7 \%$; MSRA-5, $54.2 \%$ vs $84.1 \%$; SARC-F, $91.8 \%$ vs $98.4 \%$ ), despite similar sensitivity of the instruments in the two studies. The AUC of the SARC-CalF in this study (0.734) was slightly lower than the previously reported AUC, which ranged from 0.736 to 0.92 according to different race and diagnostic criteria. ${ }^{17-19}$
Screening for sarcopenia using $\mathrm{CC}$ alone is more accurate and easier than with other widely used screening tools. This study found the accuracy of the CC method for predicting sarcopenia is significantly better than other screening tools, as shown by the significantly larger AUC of the $\mathrm{CC}$ method and the fact that $\mathrm{CC}$ remained independently associated with sarcopenia after adjusting for common covariates including the SARC-CalF score. Mo et al also found that CC was better than SARC-F and SARC-CalF for screening sarcopenia in community-dwelling Chinese older adults. ${ }^{31}$ Moreover, measuring $\mathrm{CC}$ alone is the most convenient and inexpensive method of screening for sarcopenia and can be performed by everybody. SARC-F is a well-known screening tool for sarcopenia with proven efficacy for predicting quality of life, functional impairment, hospitalization, and mobility. ${ }^{32,33}$ However, the low sensitivity (10.9\%) of SARC-F found in this study is compatible with results of a recent meta-analysis. ${ }^{8}$ In screening tools, low sensitivity may cause many missed diagnoses of people who do in fact have a particular condition. Of the screening tools evaluated for this study, the CC model had highest sensitivity and moderate specificity. Diagnostic accuracy of SARC-F and MSRA-5 found in this study for

Table 3 Performance of Four Screening Tools for Sarcopenia Defined by AWGS 2019 Criteria

\begin{tabular}{|l|l|l|l|l|l|}
\hline & Sensitivity (\%) & Specificity (\%) & +LR & -LR & AUC \\
\hline SARC-F & $10.9(5.3-19.1)$ & $91.8(83.8-96.6)$ & $1.32(0.5-3.3)$ & $0.97(0.9-1.1)$ & $0.576(0.500-0.650)$ \\
MSRA-5 & $60.7(49.7-70.9)$ & $54.2(42.9-65.2)$ & $1.33(1.0-1.8)$ & $0.73(0.5-1.0)$ & $0.600(0.523-0.674)$ \\
SARC-CalF & $38.0(28.1-48.8)$ & $80.0(69.9-87.9)$ & $1.9(1.2-3.1)$ & $0.77(0.6-0.9)$ & $0.734(0.663-0.798)$ \\
CC & $80.4(70.9-88.0)$ & $71.8(61.0-81.0)$ & $2.85(2.0-4.1)$ & $0.27(0.2-0.4)$ & $0.819(0.754-0.872)$ \\
\hline
\end{tabular}

Abbreviations: AWGS, Asian Working Group for Sarcopenia; +LR, positive likelihood ratio; -LR, negative likelihood ratio; AUC, area under the curve; MSRA-5, 5-item Mini Sarcopenia Risk Assessment; CC, calf circumference. 
predicting sarcopenia is in agreement with previous studies that recruited community-dwelling older adults. ${ }^{24}$ The finding of this study that the diagnostic accuracy of SARC-CalF was better than that of MSRA-5 or SARC-F is compatible with previous studies of older adults living in communities and nursing homes. ${ }^{18,20}$

The optimal cutoff value for CC to predict sarcopenia or skeletal muscle mass may vary according to regional and ethnic differences. Reported CC cutoff values have ranged from $32 \mathrm{~cm}$ to $34 \mathrm{~cm}$ for men and from 32 to 33 in women. The AWGS 2019 consensus recommended screening cutoff values were $<34 \mathrm{~cm}$ for men and $<33 \mathrm{~cm}$ for women ${ }^{2}$ which is in agreement with the optimal cutoff values of $\mathrm{CC}$ found in this study. The sensitivity, specificity and the AUC of CC for predicting sarcopenia in this study were consistent with results of a previous study of community-dwelling older adults in Korea, which recorded an optimal cutoff value of $32 \mathrm{~cm}$ for predicting sarcopenia in both men (sensitivity 75\%, specificity $83 \%$, AUC 0.82) and women (sensitivity $85 \%$, specificity $57 \%$, AUC 0.72). ${ }^{34}$ Further workups including measurement of muscle mass, strength and physical function should be arranged to diagnose sarcopenia if older adults were screened positive.

The independent association between BMI and sarcopenia found in this study must be interpreted with caution. Recent systematic review and meta-analysis studies found only malnutrition, instead of age, sex and BMI, was independently associated with ESGSOP-defined sarcopenia in nursing home residents. ${ }^{9}$ The findings of this study mostly agree, except for the relationship between BMI and sarcopenia. Another study found higher BMI had a protective effect against the incidence of sarcopenia in community-dwelling Chinese older adults. ${ }^{35}$ The inconsistent relationship between BMI and sarcopenia might result from differences in the characteristics of the study participants. Obesity is the most important characteristic that might affect the relationship between BMI and sarcopenia in certain studies. Although BMI is usually treated as an indicator of obesity instead of muscle mass, some studies indicate that body fat and lean mass cannot be accurately estimated by BMI. ${ }^{36}$ In contrast to $\mathrm{BMI}, \mathrm{CC}$ mainly reflects muscle mass and subcutaneous fat, and low $\mathrm{CC}$ was found to be more effective than BMI for predicting emerging care-need and mortality risk in older Chinese adults. ${ }^{37,38}$ Reports from the World Health Organization also suggest that $\mathrm{CC}$ is the most sensitive measure of muscle mass and is better than BMI in older people. $^{39}$ This study did not purposely recruit participants with a wide range of BMI values. The median BMI of the participants in this study was 23.6 (IQR, 21.3-26.0) kg/m² which falls into the categories of normal weight and overweight. Therefore, the significant association between BMI and sarcopenia found in this study cannot be extrapolated to patients who are obese or underweight. In other words, BMI might be largely influenced by body fat and may lose its ability to predict sarcopenia in individuals with high visceral fat.

There are several caveats to the findings of this study. First, due to small sample size, we did not perform subgroup analysis stratified by sex to compare the performance of four screening tools. Second, measurement of skeletal muscle mass using BIA is an inexpensive but less accurate method as compared to "gold standard" devices such as dual-energy x-ray absorptiometry. However, BIA is recommended as an alternative method by the AWGS. ${ }^{2}$ Finally, this cross-sectional study lacks direct evidence of a causal relationship between sarcopenia and CC. Further longitudinal study should be done to investigate whether $\mathrm{CC}$ could be a marker for monitoring disease progression in sarcopenia.

\section{Conclusion}

For older adults living in assisted-living facilities, the CC measurement is the most accurate, easy, and inexpensive way to screen for sarcopenia among the four common screening tools. The high sensitivity of CC in predicting sarcopenia can help long-term care facilities target at-risk older adult residents for simple exercise and diet education programs.

\section{Abbreviations}

CC, calf circumference; MSRA, Mini Sarcopenia Risk Assessment; AUC, area under the receiver operating characteristic curves; EWGSOP, European Working Group on Sarcopenia in Older People; AWGS, Asian Working Group for Sarcopenia; SARC-F, strength, assistance with walking, rise from a chair, climb stairs and falls; BIA, bioelectrical impedance analysis; ASM, appendicular skeletal muscle mass; ASMI, appendicular skeletal mass index; BMI, body mass index; IQR, interquartile range; $\mathrm{ROC}$, receiver operating characteristics.

\section{Author Contributions}

All authors met the following conditions 1, 2, 3, 4 and 5 . 
1. Made a significant contribution to the work reported, whether that is in the conception, study design, execution, acquisition of data, analysis and interpretation, or in all these areas.

2. Have drafted or written, or substantially revised or critically reviewed the article.

3. Have agreed on the journal to which the article will be submitted.

4. Reviewed and agreed on all versions of the article before submission, during revision, the final version accepted for publication, and any significant changes introduced at the proofing stage.

5. Agree to take responsibility and be accountable for the contents of the article.

\section{Funding}

This study was supported by research grants from the Wang Zhan Yang Charitable Trust, the Clinical Monitoring Research Program of Chang Gung Memorial Hospital, Keelung (Grant Number CORPG2F001CORPG2F0013), and the Health Aging Research Center, Chang Gung University and Taiwan Ministry of Education's Higher Education Deep Plowing Program (Grant Number EMRPD1I0411 and EMRPD1I0501).

\section{Disclosure}

All authors declare no competing interests.

\section{References}

1. Cruz-Jentoft AJ, Bahat G, Bauer J, et al. Sarcopenia: revised European consensus on definition and diagnosis. Age Ageing. 2019;48(1):16-31. doi:10.1093/ageing/afy169

2. Chen LK, Woo J, Assantachai P, et al. Asian working group for sarcopenia: 2019 consensus update on sarcopenia diagnosis and treatment. $J$ Am Med Dir Assoc. 2020;21(3):300-307 e302. doi:10.1016/j.jamda.2019.12.012

3. Morley JE, Anker SD, von Haehling S. Prevalence, incidence, and clinical impact of sarcopenia: facts, numbers, and epidemiology-update 2014 J Cachexia Sarcopenia Muscle. 2014;5(4):253-259. doi:10.1007/s13539014-0161-y

4. Tanimoto Y, Watanabe M, Sun W, et al. Association between muscle mass and disability in performing instrumental activities of daily living (IADL) in community-dwelling elderly in Japan. Arch Gerontol Geriatr. 2012;54 (2):e230-e233. doi:10.1016/j.archger.2011.06.015

5. Hairi NN, Cumming RG, Naganathan V, et al. Loss of muscle strength, mass (sarcopenia), and quality (specific force) and its relationship with functional limitation and physical disability: the concord health and ageing in men project. J Am Geriatr Soc. 2010;58(11):2055-2062.

6. Newman AB, Kupelian V, Visser M, et al. Strength, but not muscle mass, is associated with mortality in the health, aging and body composition study cohort. J Gerontol a Biol Sci Med Sci. 2006;61 (1):72-77. doi:10.1093/gerona/61.1.72
7. Ida S, Murata K, Nakadachi D, et al. Development of a Japanese version of the SARC-F for diabetic patients: an examination of reliability and validity. Aging Clin Exp Res. 2017;29(5):935-942. doi:10.1007/s40520-016-0668-5

8. Ida S, Kaneko R, Murata K. SARC-F for screening of sarcopenia among older adults: a meta-analysis of screening test accuracy. J Am Med Dir Assoc. 2018;19(8):685-689. doi:10.1016/j.jamda.2018.04.001

9. Shen Y, Chen J, Chen X, Hou L, Lin X, Yang M. Prevalence and associated factors of sarcopenia in nursing home residents: a systematic review and meta-analysis. $J$ Am Med Dir Assoc. 2019;20(1):5-13. doi:10.1016/j.jamda.2018.09.012

10. Rossi AP, Micciolo R, Rubele S, et al. Assessing the risk of sarcopenia in the elderly: the Mini Sarcopenia Risk Assessment (MSRA) questionnaire. $J$ Nutr Health Aging. 2017;21(6):743-749. doi:10.1007/s12603-017-0921-4

11. Cruz-Jentoft AJ, Baeyens JP, Bauer JM, et al. Sarcopenia: European consensus on definition and diagnosis: report of the European Working Group on Sarcopenia in Older People. Age and Ageing. 2010;39(4):412-423. doi:10.1093/ageing/afq034

12. Lear SA, Humphries KH, Kohli S, Chockalingam A, Frohlich JJ, Birmingham CL. Visceral adipose tissue accumulation differs according to ethnic background: results of the Multicultural Community Health Assessment Trial (M-CHAT). Am J Clin Nutr. 2007;86 (2):353-359. doi:10.1093/ajen/86.2.353

13. Zeng Y, Hu X, Xie L, Han Z, Zuo Y, Yang M. The prevalence of sarcopenia in Chinese elderly nursing home residents: a comparison of 4 diagnostic criteria. J Am Med Dir Assoc. 2018;19(8):690-695. doi:10.1016/j.jamda.2018.04.015

14. Hwang A-C, Liu L-K, Lee W-J, Peng L-N, Chen L-K. Calf circumference as a screening instrument for appendicular muscle mass measurement. J Am Med Dir Assoc. 2018;19(2):182-184. doi:10.1016/j.jamda.2017.11.016

15. Kawakami R, Murakami H, Sanada K, et al. Calf circumference as a surrogate marker of muscle mass for diagnosing sarcopenia in Japanese men and women. Geriatr Gerontol Int. 2015;15 (8):969-976. doi:10.1111/ggi.12377

16. Nishioka S, Yamanouchi A, Matsushita T, Nishioka E, Mori N, Taguchi S. Validity of calf circumference for estimating skeletal muscle mass for Asian patients after stroke. Nutrition. 2020;111028. doi:10.1016/j.nut.2020.111028

17. Barbosa-Silva TG, Menezes AMB, Bielemann RM, Malmstrom TK, Gonzalez MC, Grupo de Estudos em Composicao Corporal e N. Enhancing SARC-F: improving sarcopenia screening in the clinical practice. J Am Med Dir Assoc. 2016;17(12):1136-1141. doi:10.1016/ j.jamda.2016.08.004

18. Yang $\mathrm{M}, \mathrm{Hu} \mathrm{X}$, Xie $\mathrm{L}$, et al. Screening sarcopenia in community-dwelling older adults: SARC-F vs SARC-F combined with calf circumference (SARC-calF). J Am Med Dir Assoc. 2018;19(3):277e271-277 e278. doi:10.1016/j.jamda.2017.12.016

19. Urzi F, Simunic B, Buzan E. Basis for sarcopenia screening with the SARC-CalF in nursing homes. J Am Med Dir Assoc. 2017;18 (11):991e995-991 e910. doi:10.1016/j.jamda.2017.07.011

20. Yang M, Lu J, Jiang J, Zeng Y, Tang H. Comparison of four sarcopenia screening tools in nursing home residents. Aging Clin Exp Res. 2019;31(10):1481-1489. doi:10.1007/s40520-018-1083-x

21. Borson S, Scanlan J, Brush M, Vitaliano P, Dokmak A. The mini-cog: a cognitive 'vital signs' measure for dementia screening in multi-lingual elderly. Int J Geriatr Psychiatry. 2000;15(11):1021-1027. doi:10.1002/ 1099-1166(200011)15:11<1021::AID-GPS234>3.0.CO;2-6

22. Chen LK, Liu LK, Woo J, et al. Sarcopenia in Asia: consensus report of the Asian working group for sarcopenia. J Am Med Dir Assoc. 2014;15(2):95-101. doi:10.1016/j.jamda.2013.11.025

23. Berg HE, Tedner B, Tesch PA. Changes in lower limb muscle cross-sectional area and tissue fluid volume after transition from standing to supine. Acta Physiol Scand. 1993;148(4):379-385. doi:10.1111/j.1748-1716.1993.tb09573.x 
24. Yang M, Hu X, Xie L, et al. Comparing mini sarcopenia risk assessment with SARC-F for screening sarcopenia in community-dwelling older adults. J Am Med Dir Assoc. 2019;20(1):53-57. doi:10.1016/j. jamda.2018.04.012

25. Woo J, Leung J, Morley JE. Validating the SARC-F: a suitable community screening tool for sarcopenia? J Am Med Dir Assoc. 2014;15(9):630-634. doi:10.1016/j.jamda.2014.04.021

26. DeLong ER, DeLong DM, Clarke-Pearson DL. Comparing the areas under two or more correlated receiver operating characteristic curves: a nonparametric approach. Biometrics. 1988;44(3):837-845. doi: $10.2307 / 2531595$

27. Youden WJ. Index for rating diagnostic tests. Cancer. 1950;3 (1):32-35.

28. Cohen JF, Korevaar DA, Altman DG, et al. STARD 2015 guidelines for reporting diagnostic accuracy studies: explanation and elaboration. BMJ Open. 2016;6(11):e012799. doi:10.1136/bmjopen-2016-012799

29. Hajian-Tilaki K. Receiver Operating Characteristic (ROC) curve analysis for medical diagnostic test evaluation. Caspian $J$ Intern Med. 2013;4(2):627-635.

30. Leeflang MM, Rutjes AW, Reitsma JB, Hooft L, Bossuyt PM. Variation of a test's sensitivity and specificity with disease prevalence. CMAJ. 2013;185(11):E537-E544. doi:10.1503/cmaj.121286

31. Mo YH, Zhong J, Dong X, et al. Comparison of three screening methods for sarcopenia in community-dwelling older persons. $J \mathrm{Am}$ Med Dir Assoc. 2020. doi:10.1016/j.jamda.2020.05.041

32. Wu TY, Liaw CK, Chen FC, Kuo KL, Chie WC, Yang RS. Sarcopenia screened with SARC-F questionnaire is associated with quality of life and 4-year mortality. J Am Med Dir Assoc. 2016;17 (12):1129-1135. doi:10.1016/j.jamda.2016.07.029
33. Malmstrom TK, Miller DK, Simonsick EM, Ferrucci L, Morley JE. SARC-F: a symptom score to predict persons with sarcopenia at risk for poor functional outcomes. J Cachexia Sarcopenia Muscle. 2016;7 (1):28-36. doi:10.1002/jcsm. 12048

34. Kim S, Kim M, Lee Y, Kim B, Yoon TY, Won CW. Calf circumference as a simple screening marker for diagnosing sarcopenia in older Korean adults: the Korean Frailty and Aging Cohort Study (KFACS). J Korean Med Sci. 2018;33(20).

35. Yu R, Wong M, Leung J, Lee J, Auyeung TW, Woo J. Incidence, reversibility, risk factors and the protective effect of high body mass index against sarcopenia in community-dwelling older Chinese adults. Geriatr Gerontol Int. 2014;14(Suppl 1):15-28. doi:10.1111/ ggi. 12220

36. Gonzalez MC, Correia M, Heymsfield SB. A requiem for BMI in the clinical setting. Curr Opin Clin Nutr Metab Care. 2017;20 (5):314-321. doi:10.1097/MCO.0000000000000395

37. Tsai AC, Chang TL. The effectiveness of BMI, calf circumference and mid-arm circumference in predicting subsequent mortality risk in elderly Taiwanese. Br J Nutr. 2011;105(2):275-281. doi:10.1017/ S0007114510003429

38. Hsu WC, Tsai AC, Wang JY. Calf circumference is more effective than body mass index in predicting emerging care-need of older adults - results of a national cohort study. Clin Nutr. 2016;35 (3):735-740. doi:10.1016/j.clnu.2015.05.017

39. World Health Organization. Physical status: the use and interpretation of anthropometry. Report of a WHO Expert Committee.
Clinical Interventions in Aging

\section{Publish your work in this journal}

Clinical Interventions in Aging is an international, peer-reviewed journal focusing on evidence-based reports on the value or lack thereof of treatments intended to prevent or delay the onset of maladaptive correlates of aging in human beings. This journal is indexed on PubMed Central, MedLine, CAS, Scopus and the Elsevier

\section{Dovepress}

Bibliographic databases. The manuscript management system is completely online and includes a very quick and fair peer-review system, which is all easy to use. Visit http://www.dovepress.com/ testimonials.php to read real quotes from published authors. 